\title{
Adaptive optics imaging of low and intermediate redshift quasars ${ }^{\star}$
}

\author{
I. Márquez ${ }^{1}$, P. Petitjean ${ }^{2,3}$, B. Théodore ${ }^{4,5}$, M. Bremer ${ }^{6}$, G. Monnet $^{7}$, and J.-L. Beuzit ${ }^{8}$ \\ 1 Instituto de Astrofísica de Andalucía (CSIC), Apdo. 3004, 18080 Granada, Spain \\ 2 Institut d'Astrophysique de Paris, CNRS, 98bis Bd Arago, 75014 Paris, France \\ 3 UA CNRS 173 - DAEC, Observatoire de Paris-Meudon, 92195 Meudon Cedex, France \\ 4 Service d'Aéronomie du CNRS, BP 3, 91371 Verrière le Buisson, France \\ 5 ACRI, 260 route du Pin Montard, BP 234, 06904 Sophia-Antipolis, France \\ 6 Bristol Univ. (Dept. of Physics) H H Wills Physics Laboratory, Tyndall Av, Bristol BS8 1TL, UK \\ 7 European Southern Observatory, Karl Schwarschild Straße 2, 85748 Garching-bei-München, Germany \\ 8 Canada-France-Hawaii Telescope Corporation, 65-1238 Mamaloha Highway, Kamuela, HI 96743, USA
}

Received 26 January 2001 / Accepted 8 March 2001

\begin{abstract}
We present the results of adaptive-optics imaging in the $H$ and $K$ bands of 12 low and intermediate redshift $(z<0.6)$ quasars using the PUEO system mounted on the Canada-France-Hawaii telescope. Five quasars are radio-quiet and seven are radio-loud. The images, obtained under poor seeing conditions, and with the QSOs $\left(m_{V}>15.0\right)$ themselves as reference for the correction, have typical spatial resolution of $F W H M \sim 0.3$ arcsec before deconvolution. The deconvolved $H$-band image of PG $1700+514$ has a spatial resolution of 0.16 arcsec and reveals a wealth of details on the companion and the host-galaxy. Four out of the twelve quasars have close companions and obvious signs of interactions. The two-dimensional images of three of the host-galaxies unambiguously reveal bars and spiral arms. The morphology of the other objects are difficult to determine from one dimensional surface brightness profile and deeper images are needed. Analysis of mock data shows that elliptical galaxies are always recognized as such, whereas disk hosts can be missed for small disk scale lengths and large QSO contributions.
\end{abstract}

Key words. galaxies: active - galaxies: quasars - galaxies: fundamental parameters - galaxies: photometry infrared: galaxies

\section{Introduction}

Evidence that nearby bright galaxies contain massive dark objects in their center has become increasingly compelling over the last few years and early suggestions that a tight correlation exists between the mass of the dark object and the mass of the bulge (Kormendy \& Richstone 1995) have been convincingly corroborated (Magorrian et al. 1998; Ferrarese \& Merrit 2000). It is thus possible that AGN activity is a usual episode of the history of most, if not all, present-day bright galaxies. One way to investigate this is to determine the luminosity and morphology of galaxies hosting quasars. In addition, this gives clues on the range of conditions needed for strong nuclear activity to occur.

Send offprint requests to: I. Márquez,

e-mail: isabel@iaa.csic.es

* Based on data obtained at the Canada-France-Hawaii telescope which is operated by CNRS of France, NRC of Canada and the University of Hawaii.
Recent observations of host-galaxies with HST have questioned the previous belief that radio-loud and radioquiet quasars are found preferentially in the center of, respectively, elliptical and spiral galaxies (Bahcall et al. 1997; McLure et al. 1999). This supports previous conclusions from IR ground-based observations (Dunlop et al. 1993; McLeod \& Rieke 1995; Taylor et al. 1996) that the distinction in host-galaxy characteristics between the two classes of QSOs is subtle (see also Hooper et al. 1997, for larger redshift). More surprising is the finding by McLure et al. (1999) that host-galaxies of luminous AGNs (both radio-quiet and radio-loud) are all massive ellipticals whereas Bahcall et al. (1997) found that, in the same redshift range, host-galaxies are of various morphologies but that all radio-loud quasars have bright elliptical hosts or occur in interacting systems (Kirkhakos et al. 1999).

The detection and analysis of host-galaxies is difficult even from space (Bahcall et al. 1994, 1995; McLeod \& Rieke 1995; McLeod \& McLeod 2001). Indeed, the determination of the PSF and the subtraction of the point 
source image are crucial in this work. Differentiation between the two classical profiles, either an exponential disk or a de Vaucouleurs power-law, is effective only in the regions close to the center, or in the far-wings of the PSF (see Fig. 1 of McLure et al. 2000). This is the main limiting factor in determining host-galaxy morphologies from the ground. With the advent of adaptive optics, it will be possible to alleviate the seeing limitation (Stockton et al. 1998; Aretxaga et al. 1998; Hutchings et al. 1999). Observing in the infrared minimizes the difference in luminosity between the host and nucleus again improving our ability to determine the host morphology.

PSF determination is still a major problem but the difficulties are balanced by the prospect of using very soon $10 \mathrm{~m}$ class telescopes which will provide higher sensitivity and better spatial resolution.

In this paper we present the result of a pilot programme aiming at testing the capabilities of adaptive optics in this field. We present the data in Sect. 2, discuss each object in Sect. 3, analyze the results in Sect. 4 and conclude in Sect. 5.

\section{Sample selection and data}

In order to use adaptive optics correction quasars were selected such that the nuclei were bright enough to be used as the wavefront reference point source. The sample of radio-quiet quasars were all $\mathrm{PG}$ quasars with $m_{\mathrm{b}}<16.5$ and with redshift less than 0.6 . The radio-loud objects were selected from $3 \mathrm{C}, 4 \mathrm{C}, \mathrm{B} 2$ and PKS catalogues with the same magnitude and $z$ criteria. The final objects observed (see Table 1) were selected based upon the suitability for the observing conditions on the observing runs.

We used the CFHT adaptive optics bonnette (PUEO) and the IR camera KIR on May 1998 (run 1) and May 1999 (run 2). The weather conditions were poor during both runs and the $F W H M$ of the seeing PSF was never better than 0.8 arcsec. The adaptative-optics correction was performed on the QSOs themselves. The quasar was centered successively in the center of the four quadrants of the detector. The exposure time for individual images was two minutes. The background was determined by medianaveraging the frames and the flat-field was taken to be the normalized dark-substracted background. The images were then aligned and added. The final images have a typical resolution of $F W H M \sim 0.3$ arcsec. After each science observation an image of a star with similar magnitude as the QSO was taken in order to determine the PSF and use it to deconvolve the images. Due to rapid variations in the wheather conditions however, it was not always possible to follow this predefined procedure.

A synthetic PSF function, derived from the stellar images was used to deconvolve each of the images. As it was not always possible to apply a standard procedure due to fluctuating seeing conditions, a careful, although somewhat arbitrary choice of the PSF had to be done. In Fig. 2 we show the images of PKS $1700+514$ obtained using, for the deconvolution, three different PSFs from stars observed during the same night. These have respectively, $F W H M=0.30,0.42$ and 0.48 arcsec. The initial image of the object has a resolution of $F W H M=0.26$ arcsec and the star observed just after the science exposure has $F W H M=0.48$ arcsec. It is apparent that the best result is obtained using the star with the $F W H M$ closest to that of the science exposure. Here, we were guided in the exercice by the existence of the HST image by Hines et al. (1999). In general, this illustrates the crucial role played by a careful PSF determination in AO observations.

Results are summarized in Table 2. Columns \#3 and \#4 give, respectively, the number of objects (probably companions) found within 5 and 10 arcsec from the quasar down to $m_{H}=20.5$; columns $\# 5$ and $\# 6$ give the maximum radial distance (in arcsec and kilo-parsec) to which the host is detected at a significance level of $3 \sigma$ above the background; column \#7 gives the total magnitude of the object in the $H$-band and columns \#8 and \#9 those of the host-galaxy as derived from the PSF subtraction and profile fitting respectively (see Sect. 4); the assigned morphology, which comes from the $2 \mathrm{D}$ brightness distribution and the comparison of the two profile fittings, is given in column \#10.

\section{Comments on individual objects}

\subsection{PKS 1302-102}

The image obtained at CFHT under moderately good seeing conditions is of similar quality to that obtained with HST by Bahcall et al. (1995) (see for comparison Hutchings et al. 1994) with $F W H M=0.24$ arcsec after deconvolution (see Fig. 1). The two objects at 1 and 2 arcsec from the quasar are well-detached, and are more clearly seen when both the PSF and a model for the host-galaxy (obtained by masking the companions and fitting ellipses to the isophotes) are subtracted. It is unlikely that these companions are intervening objects as strong associated metal line absorption would be expected at such a small impact parameter when no such absorption is detected in the HST spectrum down to $w_{\text {obs }} \sim 0.2 \AA \quad$ (Jannuzi et al. 1998). The host-galaxy of this quasar has been detected by HST and fitted with a $r^{1 / 4}$ profile (Disney et al. 1995). Márquez et al. (1999) derive that the galaxy contributes $40 \%$ the total flux in the $J$-band when McLeod \& Rieke (1994) measure this contribution to be $31 \%$ of the total flux in the $H$-band after fitting an exponential profile to the host-galaxy. We have performed a similar fit on the present data and found that the contribution of the galaxy amounts to $39 \%$ in $H$ and $18 \%$ in $K$ (see Table 2). However, Fig. 4 shows that an $r^{1 / 4}$ profile is a better fit. In that case, the contribution of the host-galaxy to the total light is $70 \%(60 \%)$ in $H(\mathrm{~K})$, in good agreement with the values derived by subtracting a scaled version of the PSF and directly integrating the residual flux. 
Table 1. Log of the observations

\begin{tabular}{|c|c|c|c|c|c|c|}
\hline Name & Other names & $m_{v}$ & $z$ & $\operatorname{run}^{1}$ & $\begin{array}{l}\text { Exposure } \\
\text { (minutes) }\end{array}$ & $\begin{array}{c}F W H M^{2} \\
(\operatorname{arcsec})\end{array}$ \\
\hline Q $0955+326$ & $3 \mathrm{C} 232$ & 15.8 & 0.530 & 1 & 20 & 0.36 \\
\hline PG $1001+291$ & & 16.0 & 0.329 & 1 & 60 & 0.24 \\
\hline PG $1012+008$ & & 15.6 & 0.185 & 1 & 20 & 0.26 \\
\hline PKS 1302-102 & & 15.2 & 0.286 & 1,2 & 20,48 & $0.26,0.42$ \\
\hline PG $1402+261$ & & 15.5 & 0.164 & 1,2 & 40,64 & $0.26,0.34$ \\
\hline B2 $1425+26$ & & 15.7 & 0.366 & 1,2 & 35,64 & $0.24,0.34$ \\
\hline Q $1618+177$ & 3C 334 & 16.0 & 0.555 & 1 & 30 & 0.36 \\
\hline PG $1700+518$ & & 15.1 & 0.290 & 1,2 & 40,40 & $0.26,0.30$ \\
\hline Q $1704+608$ & $3 \mathrm{C} 351$ & 15.3 & 0.371 & 1,2 & 40,64 & $0.26,0.43$ \\
\hline B2 $1721+34$ & & 15.4 & 0.206 & 1 & 75 & 0.24 \\
\hline PG $2112+059$ & & 15.6 & 0.466 & 1,2 & 30,32 & $0.24,0.34$ \\
\hline PKS 2128-12 & & 16.2 & 0.501 & 2 & $24^{a}$ & $0.61,0.48$ \\
\hline
\end{tabular}

Table 2. Characteristics of host-galaxies

\begin{tabular}{|c|c|c|c|c|c|c|c|c|c|}
\hline \multirow[t]{2}{*}{ Name } & \multirow[t]{2}{*}{$z$} & \multicolumn{2}{|c|}{ Companions } & \multicolumn{2}{|c|}{ Extension } & \multirow{2}{*}{$m_{\mathrm{T}}^{H}\left(m_{\mathrm{T}}^{K}\right)$} & \multirow[t]{2}{*}{$m_{\mathrm{PSFsub}}^{H}\left(m_{\mathrm{PSFsub}}^{K}\right)$} & \multirow[t]{2}{*}{$m_{r^{1 / 4}}^{H}\left(m_{r^{1 / 4}}^{K}\right)$} & \multirow[t]{2}{*}{ Host type } \\
\hline & & $5 \operatorname{arcsec}$ & $10 \operatorname{arcsec}$ & $\operatorname{arcsec}$ & $\mathrm{kpc}^{a}$ & & & & \\
\hline Q $0955+326$ & 0.530 & & & 2.2 & 7.9 & 14.18 & 14.25 & 12.56 & $\mathrm{E}$ \\
\hline PG $1001+291$ & 0.329 & & & 2.9 & 8.1 & 13.87 & 14.10 & 14.84 & $\mathrm{SBa}$ \\
\hline PG $1012+008$ & 0.185 & 1 & 1 & 3.0 & 6.3 & 16.71 & 16.97 & 17.19 & E-Sa \\
\hline PKS 1302-102 & 0.286 & 2 & & 2.6 & 7.1 & $13.45(12.53)$ & $13.84(12.89)$ & $13.84(13.08)$ & $\mathrm{E}$ \\
\hline PG $1402+261$ & 0.164 & & & 3.0 & 5.3 & $13.23(11.91)$ & $14.07(12.86)$ & 14.07 (11.67) & $\mathrm{SBa}$ \\
\hline B2 $1425+26$ & 0.366 & & 2 & 2.3 & 7.5 & $14.26(13.30)$ & $14.69(14.01)$ & $15.01(14.23)$ & E-Sa \\
\hline Q $1618+177$ & 0.555 & 1 & 2 & 1.5 & 5.4 & 14.68 & 15.18 & 15.48 & \\
\hline PG $1700+518$ & 0.290 & 1 & & 2.5 & 6.7 & $12.90(11.75)$ & $13.89(12.50)$ & $13.89(12.15)$ & Sa? \\
\hline Q $1704+608$ & 0.371 & & 1 & 2.3 & 6.9 & $13.30(12.21)$ & $14.37(12.89)$ & $14.30(12.84)$ & $\mathrm{E}$ \\
\hline B2 $1721+34$ & 0.206 & & 2 & 1.5 & 3.2 & 13.95 & 14.13 & 15.07 & \\
\hline PG $2112+059$ & 0.466 & 1 & 1 & 2.0 & 6.6 & $13.64(12.64)$ & $14.10(12.96)$ & $14.71(13.51)$ & $\mathrm{E} ?$ \\
\hline PKS 2128-12 & 0.501 & & 1 & 1.7 & 6.1 & $13.42(12.77)$ & $14.39(13.33)$ & $14.50(13.21)$ & \\
\hline
\end{tabular}

${ }^{a} H_{0}=100 \mathrm{~km} \mathrm{~s}^{-1} \mathrm{Mpc}^{-1} ; q_{\mathrm{o}}=0.5$.

\section{2. $P G 1700+514$}

PG $1700+514$ is one of the most infrared-luminous, radioquiet BAL quasars (Turnshek et al. 1985, 1997). Groundbased imaging revealed an extension about 2 arcsec northeast of the quasar (Stickel et al. 1995) which was shown by adaptive-optics imaging and follow-up spectroscopy to be a companion with a redshift $140 \mathrm{~km} \mathrm{~s}^{-1}$ blueward of the quasar (Stockton et al. 1998). NICMOS observations lead Hines et al. (1999) to argue that the companion is a collisionally induced ring galaxy. The fit to the SED and the Keck spectrum of the companion imply that the light is emitted by an old population of stars plus a $85 \mathrm{Myr}$ old star-burst (Canalizo \& Stockton 1997). Note however that the $H$-band flux $\left(m_{H} \sim 16.6\right)$ deduced from HST imaging is much larger than that predicted by the model. Stockton et al. (1998) showed that the inclusion of embedded dust can produce a spectral-energy distribution that is consistent with both the optical spectrophotometry and the IR photometry.

The image obtained at CFHT is shown in Fig. 2. We confirm the findings by Stockton et al. (1998) that the companion has the appearance of an arc with several condensations. We used different PSF to deconvolve the image. The best deconvolution is obtained using the star with the FWHM closest to that of the AGN (0.30 arcsec, see Sect. 2). The image has a final resolution of 0.16 arcsec and is probably the best image obtained yet on this object. The companion is seen as a highly disturbed system with a bright nucleus and a ring-like structure; the nucleus being decentered with respect to the ring. The host-galaxy is clearly seen around the quasar with a bright extension to the south-west, first noted by Stickel et al. (1995) and clearly visible in the optical images by Stockton et al. (1998). In addition, we detect a bright knot to the 


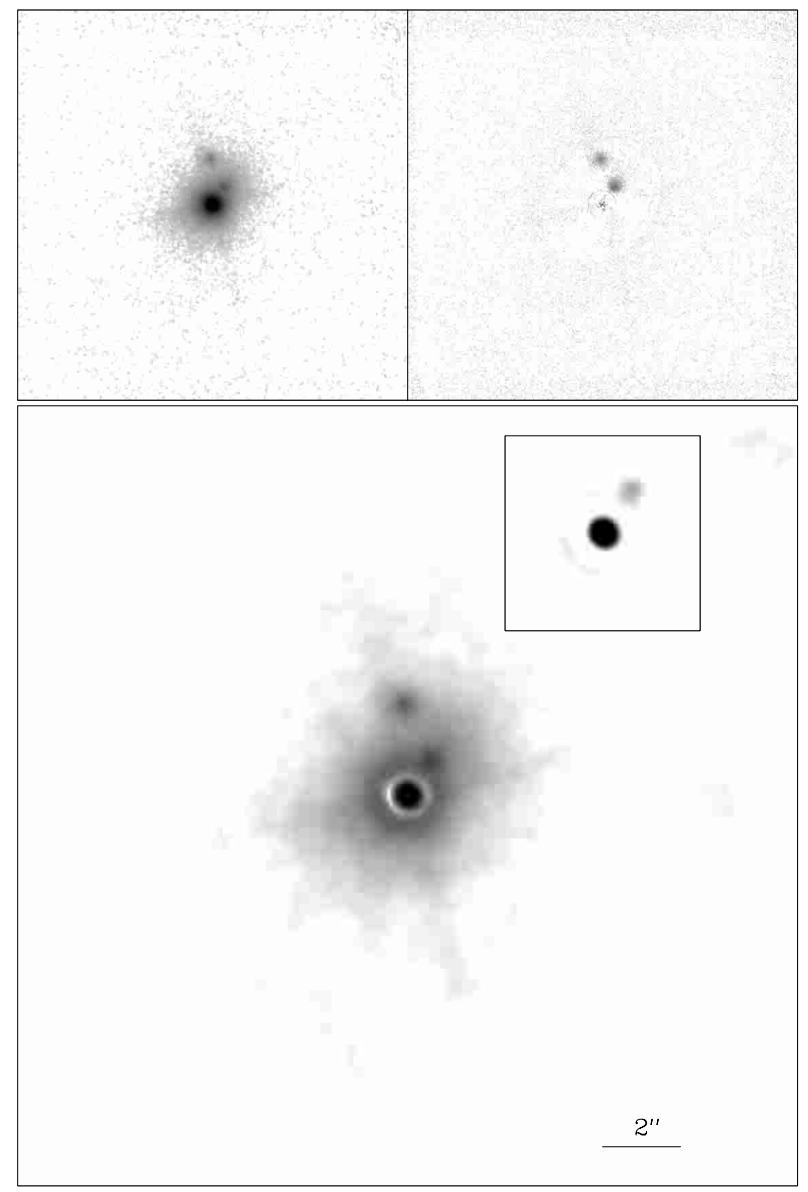

Fig. 1. Images of PKS 1302-102 in the $H$-band. The top-left panel and bottom panel correspond to the image, respectively, before and after deconvolution. The spatial resolutions are respectively $F W H M \sim 0.32$ and 0.24 arcsec. The top right panel shows the two companions after substraction of the PSF and a model for the host-galaxy. The inset in the bottom panel corresponds to a higher contrast version of the inner $4 \operatorname{arcsec} x$ 4 arcsec

south-east which is not seen in the NICMOS data probably because of the presence of residuals in the PSF subtraction. The comparison between the HST and CFHT images of PG $1700+514$ shows how powerful AO can be, and bodes well for the use of the technique on $10 \mathrm{~m}$-class telescopes. No obvious relation is found between the nearIR image and the radio map (Hutchings et al. 1992).

\subsection{Other objects}

3C 232 - TON 0469 This object has attracted much interest because it is located $2 \operatorname{arcmin}\left(\sim 8 h^{-1} \mathrm{kpc}\right.$ at $z=0.0049)$ north of the nearby $(z=0.0049)$ galaxy NGC 3067 and has been considered as the prototype of the galaxy-quasar physical association as a probe for anomalous redshifts (Hoyle \& Burbidge 1996). The HST spectrum shows a strong Mg II system at $z_{\text {abs }}=0.0049$ (Tumlinson et al. 1999) which has been shown to be due to an $\mathrm{H}$ I tidal tail originating in the disk of the

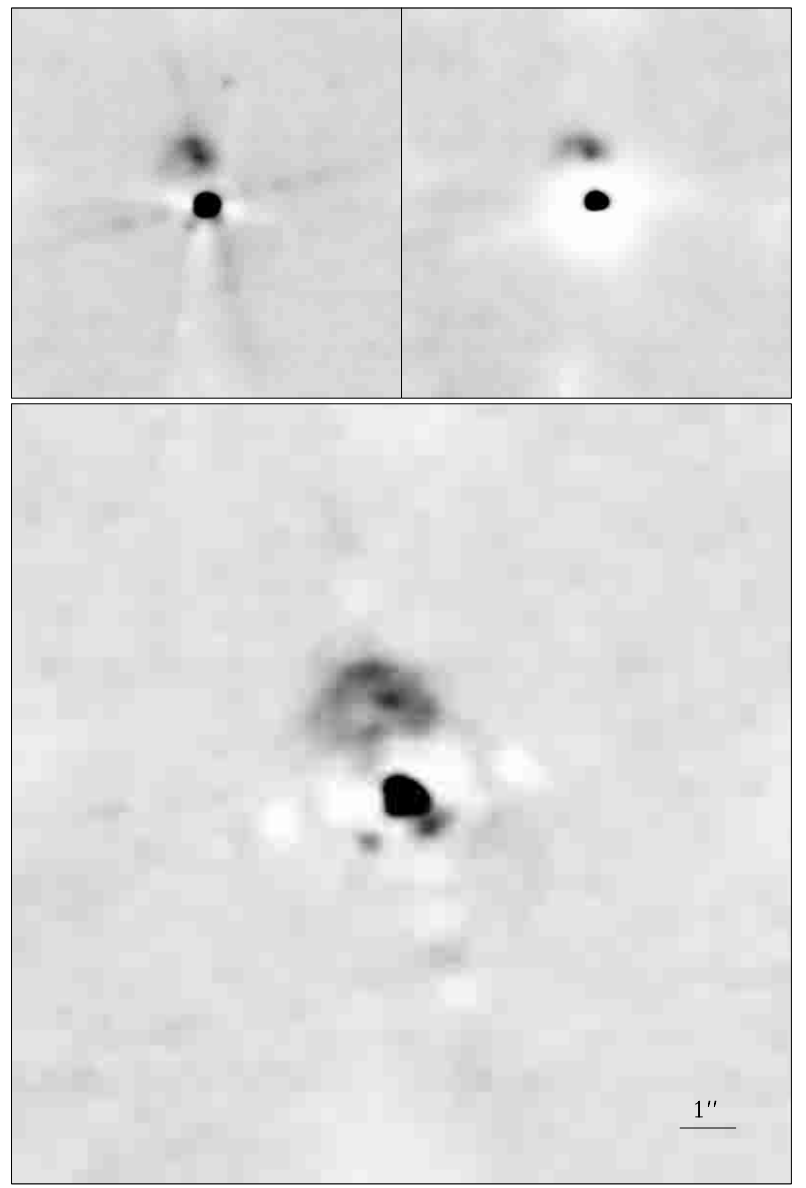

Fig. 2. Images of PG $1700+514$ after deconvolution using the PSF given by three stars observed during the same night. The best deconvolution is obtained using the star with the $F W H M$ closest to that of the quasar. The resulting image (bottom) has a final resolution of $F W H M=0.16$ arcsec

foreground galaxy (Carilli et al. 1989). The MERLIN map at $1.6 \mathrm{GHz}$ shows a slight extension to the east (Akujor et al. 1994). The host-galaxy is detected in the CFHT image with an extension to the south-west up to 5 arcsec from the quasar). From both the image (Fig. 3) and the profile fitting (Fig. 4) we derive that the host is better described as an elliptical galaxy.

PG 1001+291 - Ton 0028 Only one image from the HST archive has been published yet (Boyce et al. 1999). These authors favor the interpretation that the object is an interacting object with two nuclei, one 1.9 arcsec to the south-west and the other 2.3 arcsec to the north-east. We find no evidence for the presence of two nuclei. In particular there is no residual after subtraction of a hostgalaxy model. The profile is not well fitted by a single disk component, due to the presence of a bump which is characteristic of the bar component in the profiles of barred spirals. We therefore conclude that the elongated NE-SW feature seen in our image probably reveals the presence of a strong bar in an otherwise spiral galaxy. 
PG 1012+008 This radio-quiet quasar interacts with two companions located to the north and east (Heckman et al. 1984). It has been imaged with HST by Bahcall et al. (1997) who concluded from 2D-modelling that the host is a spiral galaxy with $r_{1 / 2}=10.8 \mathrm{kpc}$. They also fit the onedimensional profile as an elliptical with $r_{1 / 2}=24.5 \mathrm{kpc}$. The best 2D fit by McLure et al. (1999) is a large elliptical with $r_{1 / 2}=23 \mathrm{kpc}$. We find that the best fit to the $H$ band profile is a $r^{1 / 4}$ law with a scalelength of 2.9 arcsec or $6 h^{-1} \mathrm{kpc}$ (see Fig. 3).

PG 1402+261 - TON 0182 Bahcall et al. (1997) determined from HST imaging that the host of this quasar is a bright spiral galaxy with prominent $\mathrm{H}$ II regions located along the spiral arms. The CFHT image shows that there is a bright elongation extending $\sim 2$ arcsec away from the nucleus on both sides of it in the NW-SE direction, probably tracing a strong bar also seen as a bump in the surface brightness profile, see Fig. 4.

B2 1425+26 - TON 0202 This quasar is hosted by a bright galaxy probably in mild interaction with one of the three companions located within 10 arcsec from the point source and listed by Hutchings et al. (1984) and Block \& Stockton (1991). Two of these companions are detected in our $H$ and $K$-band images. The host-galaxy has been classified as elliptical by previous investigators (Malkan 1984; Kirkhakos et al. 1999). However, the isophotes in our image are distorted and an arc-like feature is clearly seen to the west (see also the F555W image of Kirkhakos et al. 1999). Moreover, an emission line nebulosity is detected by $\mathrm{H} \alpha, \mathrm{H} \beta$, [O III] emission up to 3 arcsec from the nucleus (Boroson et al. 1984; Stockton \& MacKenty 1987). Our fit shows that an elliptical galaxy (or an early spiral) is a good description for the host-galaxy (see Fig. 4).

3C 334 The host-galaxy has twisted isophotes. As noted by Lehnert et al. (1999), the galaxy appears elongated approximatively in the same direction as the radio structure (Hutchings et al. 1998). Note that the arc-like structure seen to the south in the deconvolved image was detected by its [OII] emission (Hes et al. 1996). Comparison of the new AO corrected images with previous ground-based images (e.g. Hes et al. 1996; Márquez et al. 1999) clearly shows the power of AO technique. We note that three Lyman- $\alpha$ absorptions are detected in the quasar spectrum with $w_{\text {obs }}=0.37,1.00$ and $0.21 \AA$ at redshifts $z_{\text {abs }}=0.5387,0.5449$ and 0.5491 , slightly smaller than the emission redshift $z_{\mathrm{em}}=0.555$ (Jannuzi et al. 1998). Spectroscopy of the companions is required to determine if they are somehow associated with these absorption systems. The profile fitting does not allow us to distinguish between a disk-like or an elliptical host.

3C 351 We observed this field in the $H$ and $K$-bands (see Fig. 3). The two objects closest to the line of sight in our image are situated 7 arcsec north-east of the quasar. One is a bright spiral galaxy, well resolved in our images, the other is very faint (it is barely visible in Fig. 3 but it is more clearly seen in the deconvolved image) and could be a companion of the former. Lanzetta et al. (1995) and Le Brun et al. (1996) have searched the field around 3C 351 for galaxies responsible for Lyman- $\alpha$ and C IV absorption observed by Bahcall et al. (1993) in the HST spectrum of the quasar. There are two Lyman- $\alpha$ absorption lines at $z_{\text {abs }}=0.2216$ and 0.2229 , the former showing C IV absorption as well. Le Brun et al. (1996) identified the metal line system with a galaxy $710 h_{50}^{-1} \mathrm{kpc}$ away from the line of sight. The redshift of the two objects closest to the quasar have not been determined however and it is possible that these two objects are responsible for the absorptions. If they are at $z=0.222$, the impact parameter is of the order of only $15 h^{-1} \mathrm{kpc}$. It is very important to confirm this for our understanding of the nature of $\mathrm{H} \mathrm{I}$ halos around low- $z$ galaxies. Boyce et al. (1998) detect a companion at about 3.3 arcsec east to the quasar. We do not detect this companion in any of our images although it is within the possibilities of our imaging. This may indicate that the flux in the HST-F702W filter is dominated by line emission or that the object is very blue. As there is no strong intervening absorption in the spectrum of the quasar, it is probable that this companion is at the redshift of the quasar. Note that $3 \mathrm{C} 351$ exhibits a strong associated system with $\mathrm{H}$ I Lyman- $\alpha, \mathrm{C}$ IV, $\mathrm{N}$ v and $\mathrm{O}$ VI absorptions. The presence of the associated system does not seem to be related to any other imaging property of the QSO.

B2 1721+34 The host-galaxy is detected up to 1.5 arcsec from the central point-source in the $H$-band CFHT image (see Fig. 3). The one-dimensional profile shows that a $r^{1 / 4}$ law fits the galaxy profile better than an exponential fit, but images with better $\mathrm{S} / \mathrm{N}$ ratio are needed to confirm this at higher significance.

PG 2112+059 Two companions are detected within 10 arcsec of the nucleus. An elliptical profile better describes the light distribution of the host-galaxy.

PKS 2128-12 Disney et al. (1995) fitted the one dimensional profile with a de Vaucouleurs law of radius $R_{\mathrm{e}}=37.4 \mathrm{kpc}$. The images of this object have the poorest resolution in our sample. We are therefore unable to determine a reliable fit to the host-galaxy profile. We detect a companion at 7 arcsec north-east of the nucleus.

\section{Analysis}

In each of the images, we first masked out the companion objects and the ghosts due to the telescope. We then obtained the surface brightness profiles of the galaxies using the $\mathrm{IRAF}^{1}$ task ellipse. The resulting profiles were fitted over the radius range from 3 times the $F W H M$ of the PSF

\footnotetext{
1 IRAF is the Image Analysis and Reduction Facility made available to the astronomical community by the National Optical Astronomy Observatories, which are operated by the Association of Universities for Research in Astronomy (AURA), Inc., under contract with the U.S. National Science Foundation.
} 


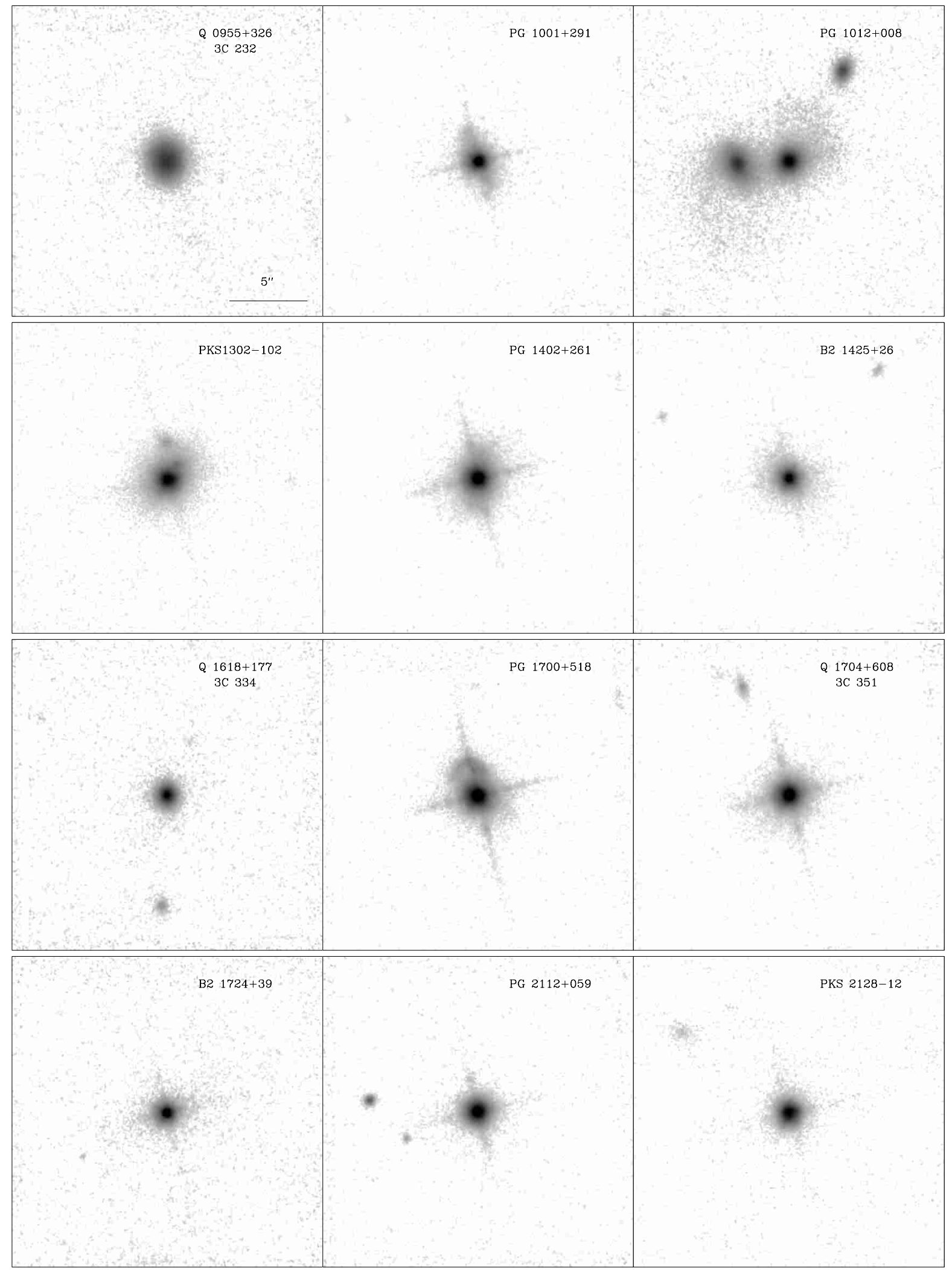

Fig. 3. Images of objects described in Sect. 3.3 and Tables 1 and 2. They are $K$ band images for PKS 1302-102 and PKS 2128-12 and $H$ band images for the rest. The scale is marked with the horizontal bar in the top-left figure and is the same for all the figures 

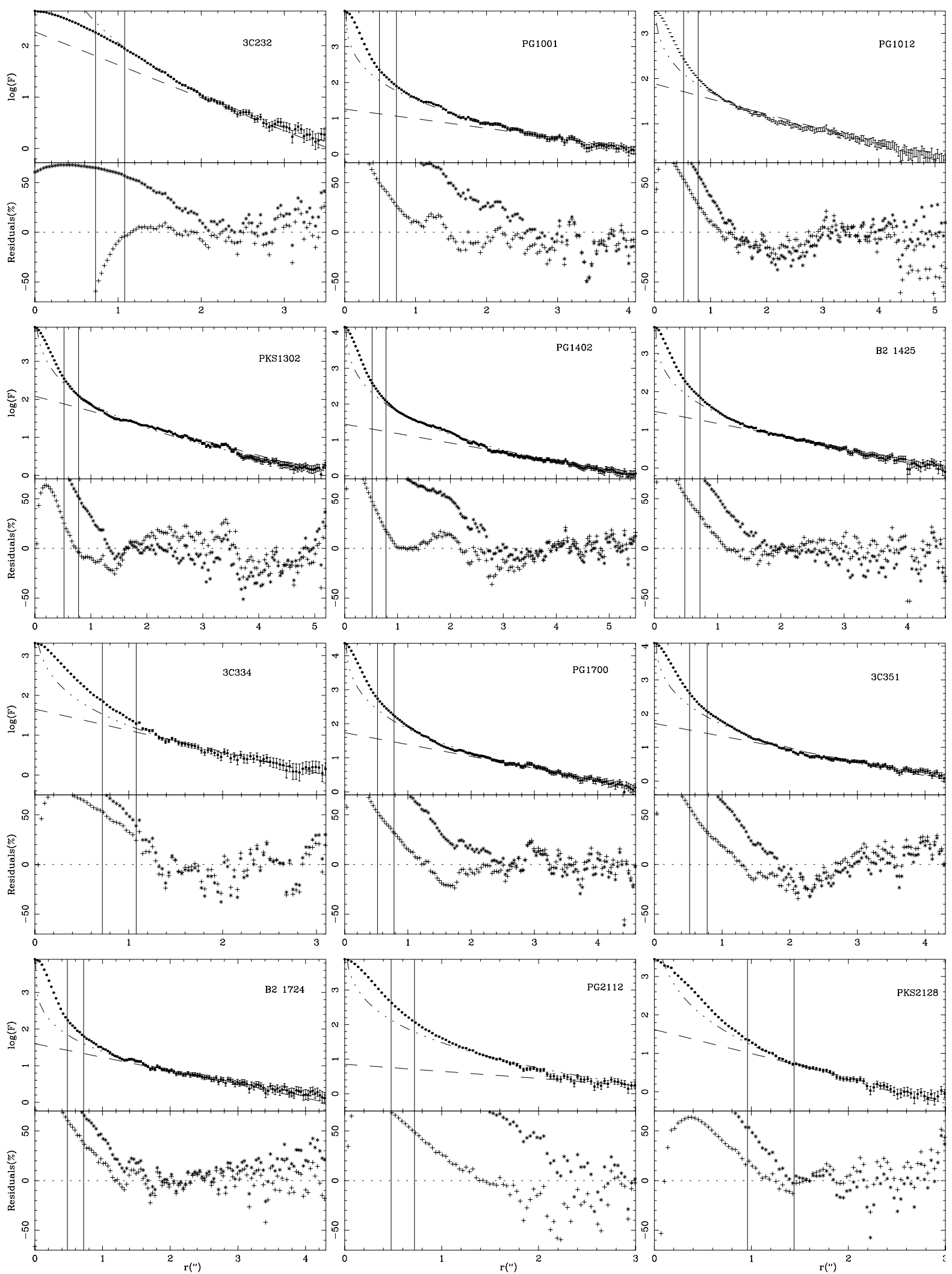

Fig. 4. Top: surface brightness profiles (dots and error bars) obtained with the undeconvolved images in the $H$-band (except for PKS 2128-12, for which we used the $K$-band image because of its better PSF). Fits by a disk (dashed line) and a $r^{1 / 4}$ law (dotted-dashed line) are overplotted. Bottom: residuals from the disk (stars) and $r^{1 / 4}$ (crosses) fits in percentage. The vertical lines are drawn at respectively two and three times the PSF FWHM 


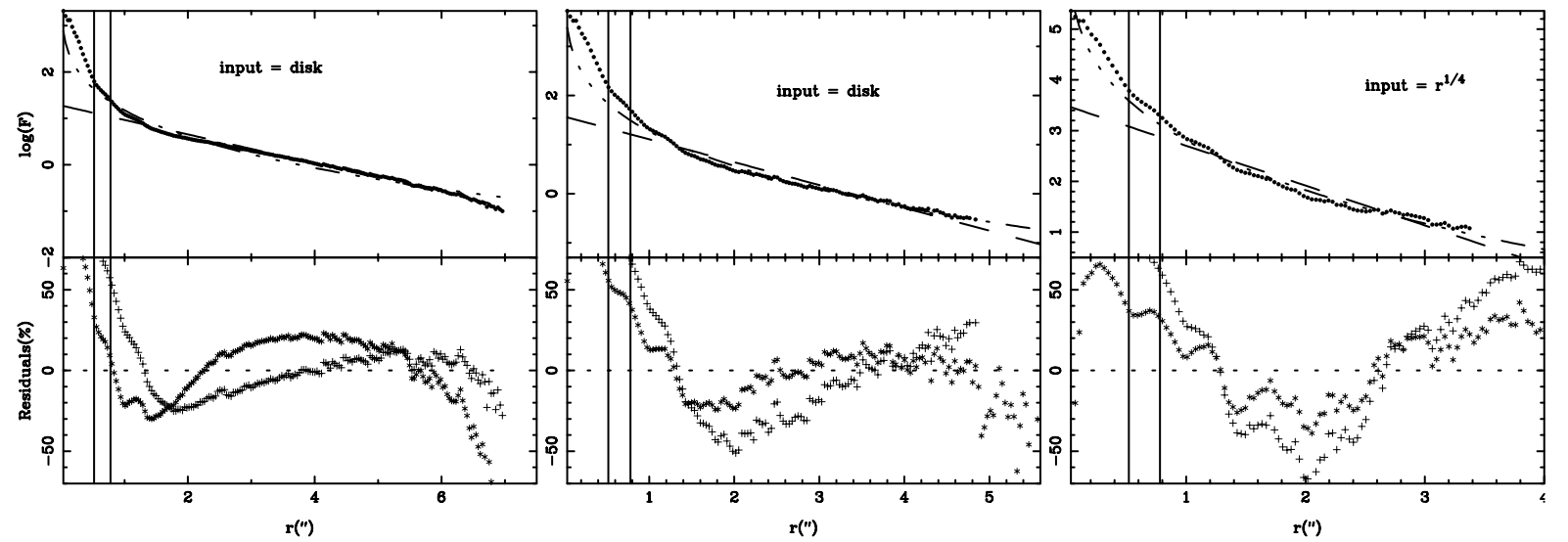

Fig. 5. Illustrative examples of mocked images and their fits. The top panel is the surface brightness profiles with models overplotted (exponential disk as a solid line and $r^{1 / 4}$ law as a dashed line). a) The input is a disk galaxy and the mocked image is better fitted by a disk host (the quasar contributes $25 \%$ to the total light). b) The input is a disk galaxy but the mocked image is equally well fitted by the two laws. The disk parameters are the same as is a), but the quasar contributes $70 \%$ to the total light. c) When the input is an elliptical galaxy, the mocked image is always better fitted by a $r^{1 / 4}$ profile

up to the point where the galaxy surface brightness level falls below $2 \times \sigma$ of the background level. We have systematically fitted an exponential disk and a de Vaucouleurs $r^{1 / 4}$ law. The results are shown in Fig. 4, where the residuals from the subtraction of the fitted profiles to the real profiles are also plotted. Out of the 10 objects for which we can extract some morphological information, two of the host-galaxies are most probably barred spirals, the rest being ellipticals or very early type spirals (see Table 2 ). We note that in general the number of points we can use to fit either profile is not large enough to unambiguously distinguish between the two fitted profiles. At small radii, the excess of light between the observed profile and the model disk can be due to the presence of a bulge. The morphology indicated in column \#10 of Table 2 is determined considering both the 2D luminosity spatial distribution and the $1 \mathrm{D}$ profile. It is apparent that to discriminate between both morphologies, the $\mathrm{S} / \mathrm{N}$ ratio must be high at large radii.

The magnitudes of the hosts have been derived by integrating the $r^{1 / 4}$ profiles for all the objects. Indeed, it can be seen in Fig. 4 that there is an excess of light at small radii compared to the disk profile for all objects. This suggests that in our sample, the disk galaxies also have a strong bulge and/or a strong bar. This is confirmed by the 2D luminosity distribution. Results are given in Table 2. We have also subtracted a scaled version of the most suitable PSF for each nucleus (imposing a non-negative profile in the center, see Márquez et al. 1999) The resulting host magnitudes, computed by integrating the PSF-subtracted images, are in good agreement with those obtained from the profile fitting (see Table 2).

In order to test our fitting procedure, we have generated images of model elliptical and disk galaxies with scale-lengths and effective surface brightness within the range derived from the data. The same orientation and axis ratio is given to all of them. A point source is added in the center of the galaxy to mimic the quasar. An appropriate amount of noise is added, and then the images are convolved with a typical observed PSF. The mock data images are analyzed in the same way as real data.

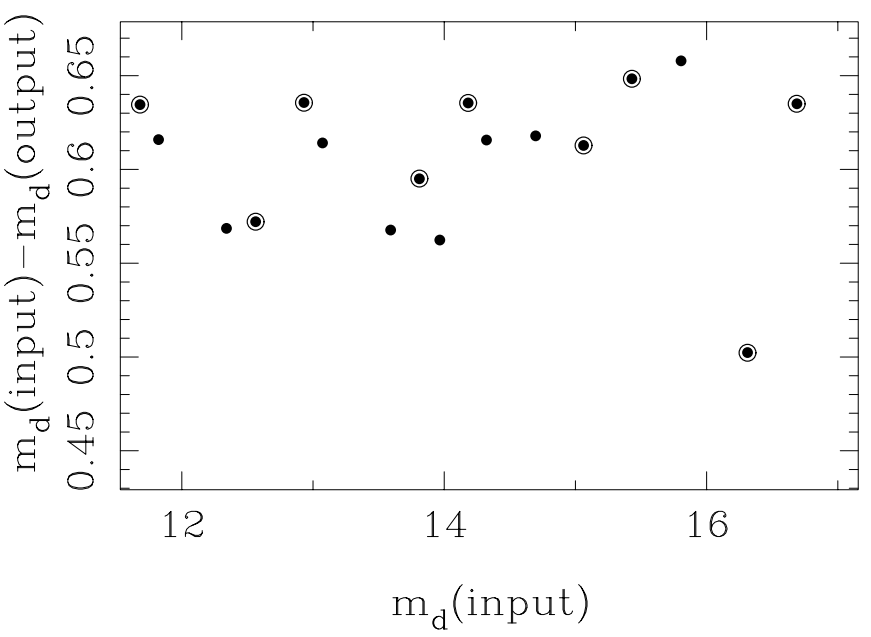

Fig. 6. Test of the fitting procedure on disk-galaxies. We plot here the difference between the input and output magnitudes versus the input magnitude in the case the host-galaxy has the same magnitude as the quasar. A dot surrounded by a circle means that the fit by an $r^{1 / 4}$ law is at least as good as the fit by an exponential disk

We first note that an elliptical galaxy is always recognized as an elliptical galaxy by the fitting procedure, whereas a disk-galaxy is better fitted by a $r^{1 / 4}$ law when the unresolved point-source contributes more than half the total light. This is illustrated in Fig. 5. This means that, at least with data of similar quality to those presented here, 


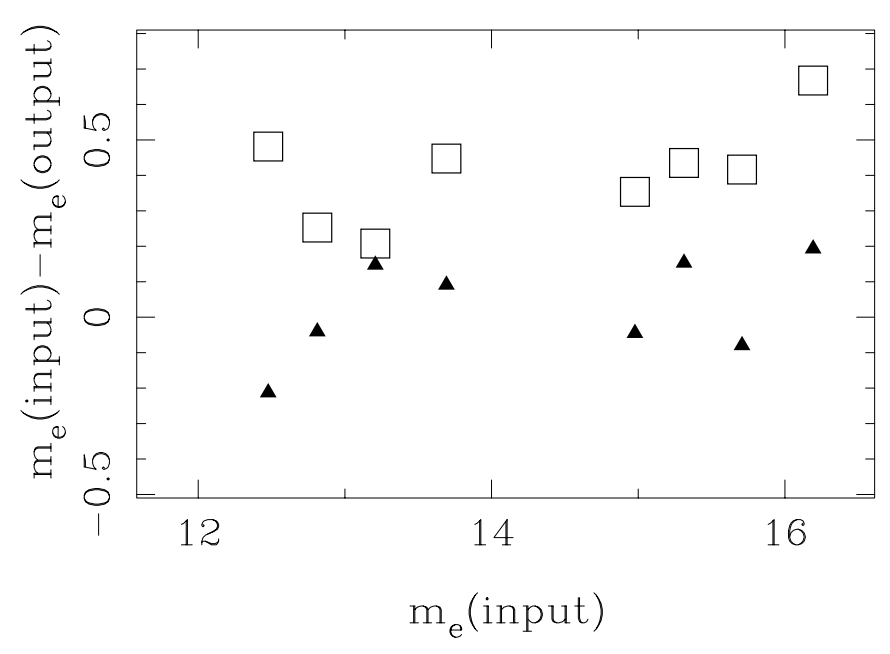

Fig. 7. Test of the fitting procedure on elliptical galaxies. We plot here the difference between the input and output magnitudes versus the input magnitude. Triangles correspond to the systems where the QSO contributes to the total luminosity as the host-galaxy does, squares represent those cases in which the host luminosity is half that of the QSO

the fraction of elliptical galaxies in the sample may be overpredicted. Going deeper, at least 0.5 to 1 mag, should help solve this problem as it is apparent that the distinction between spiral and elliptical profiles is easier when the galaxy is detected at larger distances from the central point-source.

It is interesting to note that the output magnitudes are brighter than the input in both cases, elliptical or disk galaxies (see Figs. 6 and 7). The reason for this is probably the difficulty in determining the extension of the PSF wings which, if not subtracted properly, will artificially increase the flux of the host-galaxy. In the case of spirals, the difference is as large as 0.6 mag when the contribution of the point-source is the same as the contribution of the host-galaxy (see Fig. 6). For the ellipticals, the difference is less but still important when the QSO dominates the total flux.

Note that the ratio between the QSO and the hostgalaxy luminosities is expected to increase with redshift. The above bias tends to imply that host-galaxy luminosities could be overestimated.

\section{Conclusions}

Adaptive-optics imaging in the $H$ and $K$ bands has been used to study the morphology of QSO host-galaxies at low and intermediate redshifts $(z<0.6)$. We detect the host-galaxies in 11 out of 12 quasars, of which 5 are radioquiet and 7 are radio-loud. The images, obtained under poor seeing conditions, and with the QSOs themselves as reference for the correction, have typical spatial resolution of $F W H M \sim 0.3$ arcsec before deconvolution. In the best case, the deconvolved $H$-band image of PG $1700+514$ (with a spatial resolution of 0.16 arcsec) reveals a wealth of detail on the companion and the host-galaxy, and is probably the best-quality image of this object thus far.

Four of the quasars in our sample have close companions and show obvious signs of interactions. The twodimensional images of three of the host-galaxies unambiguously reveal bars and spiral arms. For the other objects, it is difficult to determine the host-galaxy morphology on the basis of one dimensional surface brightness fits alone.

We have simulated mocked images of host-galaxies, both spirals and ellipticals, and applied the same analysis as to the data. Disk hosts can be missed for small disk scalelengths and large QSO contributions. In this case, the host-galaxy can be misidentified as an elliptical galaxy. Elliptical galaxies are always recognized as such, but with a luminosity which can be overestimated by up to 0.5 mag. The reason for this is that the method used here tends to attribute some of the QSO light to the host. This is also the case for disk galaxies with a strong contribution of the unresolved component.

Acknowledgements. We acknowledge the suggestions by the referee, Alan Stockton, that helped to improve the presentation. I. Márquez acknowledges financial support from the Spanish Ministerio de Educación y Ciencia (EX94-8826734). This work is financed by DGICyT grants PB93-0139 and PB960921. Financial support to develop the present investigation has been obtained through the Junta de Andalucía TIC-114.

\section{References}

Akujor, C. E., Lüdke, E., Browne, I. W. A., et al. 1994, A\&AS, 105,247

Aretxaga, I., Le Mignant, D., Melnick, J., Terlevich, R. J., \& Boyle, B. J. 1998, MNRAS, 298, L13

Bahcall, J. N., Bergeron, J., Boksenberg, A., et al. 1993, ApJS, 87,1

Bahcall, J. N., Kirkhakos, S., \& Schneider, D. P. 1994, ApJ, 435, L11

Bahcall, J. N., Kirkhakos, S., \& Schneider, D. P. 1995, ApJ, 450, 486

Bahcall, J. N., Kirkhakos, S., Saxe, D. H., \& Schneider, D. P. 1997, ApJ, 479, 642

Block, D. L., \& Stockton, A. 1991, AJ, 102, 1928

Boroson, T. A., Person, S. E., \& Oke, J. B. 1984, ApJ, 293, 120

Boyce, P. J., Disney, M. J., Blades, J. C., et al. 1998, MNRAS, 298, 121

Boyce, P. J., Disney, M. J., \& Bleaken, D. G. 1999, MNRAS, 302, L39

Canalizo, G., \& Stockton, A. 1997, ApJ, 480, L5

Carilli, C. L., van Gorkom, J. H., \& Stocke, J. T. 1989, Nature, 338, 134

Disney, M. J., Boyce, P. J., Blades, J. C., et al. 1995, Nature, 376, 150

Dunlop, J. S., Taylor, G. L., Hughes, D. H., \& Robson, E. I. 1993, MNRAS, 264, 455

Ferrarese, L., \& Merrit, D. 2000, ApJ, 539, L9

Heckman, T. M., Bothun, G. D., Balick, B., \& Smith, E. P. 1984, AJ, 89, 958

Hes, R., Barthel, P. D., \& Fosbury, R. A. E. 1996, A\&A, 313, 423 
Hines, D. C., Low, F. J., Thompson, R. I., Weymann, R. J., \& Storrie-Lombardi, L. J. 1999, ApJ, 512, 140

Hooper, E. J., Impey, C. D., \& Foltz, C. B. 1997, ApJ, 480, L95

Hoyle, F., \& Burbidge, G. 1996, A\&A, 309, 335

Hutchings, J. B., Morris, S. C., Gower, A. C., \& Lister, M. L. 1994, PASP, 106, 642

Hutchings, J. B., Crampton, D., \& Campbell, B. 1984, ApJ, 280, 41

Hutchings, J. B., Crampton, D., Morris, S. L., Durand, D., \& Steinbring, E. 1999, AJ, 117, 1109

Hutchings, J. B., Dewey, A., Chaytor, D., et al. 1998, PASP, 110,111

Hutchings, J. B., Neff, S. G., \& Gower, A. C. 1992, PASP, 104, 62

Jannuzi, B. T., Bahcall, J. N., Bergeron, J., et al. 1998, ApJS, 118,1

Kirkhakos, S., Bahcall, J. N., Schneider, D. P., \& Kristian, J. 1999, ApJ, 520, 67

Kormendy, J., \& Richstone, D. 1999, ARA\&A, 33, 581

Lanzetta, K. M., Bowen, D. V., Tytler, D., \& Webb, J. K. 1995, ApJ, 442, 538

Le Brun, V., Bergeron, J., \& Boissé, P. 1996, A\&A, 306, 691

Lehnert, M., Miley, G. K., Sparks, W. B., et al. 1999, ApJS, 123,351
Malkan, M. A. 1984, ApJ, 287, 555

Magorrian, J., Tremaine, S., Richstone, D., et al. 1998, AJ, 115,2285

Márquez, I., Durret, F., \& Petitjean, P. 1999, A\&AS, 135, 83

McLeod, K. K., \& McLeod, B. A. 2001, ApJ, 546, 50

McLeod, K. K., \& Rieke, G. H. 1994, ApJ, 431, 137

McLeod, K. K., \& Rieke, G. H. 1995a, ApJ, 441, 96

McLeod, K. K., \& Rieke, G. H. 1995b, ApJ, 454, L77

McLure, R. J., Dunlop, M., \& Kukula, M. J. 2000, MNRAS, 318,693

McLure, R. J., Kukula, M. J., Dunlop, M., et al. 1999, MNRAS, 308,377

Stickel, M., Fried, J. W., McLeod, K. K., \& Rieke, G. H. 1995, AJ, 109, 1979

Stockton, A., Canalizo, G., \& Close, L. M. 1998, ApJ, 500, 121

Stockton, A., \& MacKenty, J. W. 1987, ApJ, 316, 584

Taylor, G. T., Dunlop, J. S., Hughes, D. H., \& Robson, E. I. 1996, MNRAS, 283, 930

Tumlinson, J., Giroux, M. L., Shull, J. M., \& Stocke, J. T. 1999, AJ, 118, 2148

Turnshek, D. A., Foltz, C. B., Weymann, R. J., et al. 1985, ApJ, 294, L1

Turnshek, D. A., Monier, E. M., Sirola, C. J., \& Espey, B. R. 1997, ApJ, 476, 40 